\title{
Circular RNA profiling reveals circEXOC6B and circN4BP2L2 as novel prognostic biomarkers in epithelial ovarian cancer
}

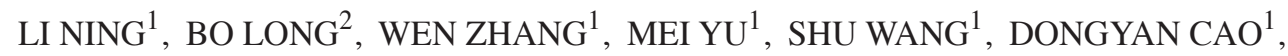 \\ JIAXIN YANG ${ }^{1}$, KENG SHEN ${ }^{1}$, YUENAN HUANG ${ }^{3}$ and JINGHE LANG ${ }^{1}$ \\ ${ }^{1}$ Department of Obstetrics and Gynecology, and ${ }^{2}$ Central Research Laboratory, Peking Union Medical College Hospital, \\ Chinese Academy of Medical Sciences and Peking Union Medical College, Beijing 100730; \\ ${ }^{3}$ Department of General Surgery, The Second Affiliated Hospital, Harbin Medical University, Harbin 150081, P.R. China
}

Received May 29, 2018; Accepted August 8, 2018

DOI: $10.3892 /$ ijo.2018.4566

\begin{abstract}
Circular RNAs (circRNAs) are regarded as a novel class of widespread endogenous non-coding RNAs, which may play important roles in tumorigenesis by regulating gene expression. Nevertheless, the characterization of circRNAs in epithelial ovarian cancer (EOC) remains largely unknown. This study aimed to investigate circRNA expression profiles in EOC. A total of 54 EOC specimens and 54 normal ovarian tissues (controls) were collected. circRNA-sequencing based circRNA expression profiles were identified in 4 EOC specimens and compared with 4 normal ovarian tissues. circRNA-sequencing data were validated by reverse transcription-quantitative polymerase chain reaction (RT-qPCR) in the 54 EOC specimens and 54 normal ovarian tissues. The association between differentially expressed circRNAs and various clinicopathological features of EOC was determined using a non-parametric test. Univariate analysis was performed using the log-rank test. A total of 4,388 circRNAs (2,556 up- and $1,832$ downregulated; fold change of $\geq 2$ and $\mathrm{P}<0.05)$ were identified to be differentially expressed in the EOC specimens compared with the normal ovarian tissues. Of these, the levels of 6 circRNAs (circBNC2, circEXOC6B, circFAM13B, circN4BP2L2, circRHOBTB3 and circCELSR1) were confirmed by RT-qPCR. Our data further indicated that these 6 circRNAs were associated with various clinicopathological features of EOC. More importantly, we found that circEXOC6B and circ-
\end{abstract}

Correspondence to: Dr Jinghe Lang, Department of Obstetrics and Gynecology, Peking Union Medical College Hospital, Chinese Academy of Medical Sciences and Peking Union Medical College, 1 Shuaifuyuan Road, Dongcheng, Beijing 100730, P.R. China E-mail: langjhpumch@126.com

Dr Yuenan Huang, Department of General Surgery, The Second Affiliated Hospital, Harbin Medical University, 246 Xuefu Road, Nangang, Harbin 150081, P.R. China

E-mail: dr-huangyuenan@163.com

Key words: epithelial ovarian cancer, circular RNA, non-coding RNA, diagnosis, prognosis
N4BP2L2 may act as novel prognostic biomarkers in patients with EOC. On the whole, the results of this study indicate that differentially expressed circRNAs may participate in the pathogenesis of EOC and may thus have potential for use as novel diagnostic and prognostic biomarkers for EOC. Future experiments with larger sample sizes are required to verify the current findings and illuminate the regulatory mechanisms of action of circRNAs in the tumorigenesis of EOC.

\section{Introduction}

Epithelial ovarian cancer (EOC) is the second most common form of gynecological cancer and is currently the first leading cause of gynecological cancer-related mortality (1). The 5-year survival rate of patients with advanced disease is merely $30 \%$ (2). Despite the intra-tumor heterogeneity, patients with EOC are usually treated with standard therapy, including cytoreductive surgery and platinum-based chemotherapy $(3,4)$. However, conventional treatment usually causes chemoresistance, resulting in tumor recurrence and even death (3). Therefore, it is imperative to identify novel molecular mechanisms and potential therapeutic targets in order to better control the severity of EOC.

Circular RNAs (circRNAs) are regarded as widespread endogenous non-coding RNAs $(5,6)$. They were discovered $>20$ years ago, but were mostly misinterpreted as mis-splicing errors due to low abundance (7). Only in 2012/2013, their substantial presence in eukaryotic cells was demonstrated using high-throughput sequencing data (8). circRNAs are characterized by covalently closed loop structures without 5' caps or 3' polyadenylated tails (9). The formation of these structures involves back-splicing events by the spliceosome at the expense of canonical mRNA isoforms $(10,11)$. Such circularization calls for the covalent binding of upstream $5^{\prime}$ acceptors to downstream 3' donors (12). Although the concrete mechanisms of back-splicing events remain unclear, flanking introns containing ALU repeats are believed to be essential for the process (13). It is becoming increasingly evident that, instead of being regarded as splicing errors, circRNAs are rather the products of regulated back-splicing events (14). Accordingly, a body of evidence has indicated that circRNAs are of huge regulatory potency (15). Previous studies have 
found that a number of circRNAs play crucial roles in human epithelial-mesenchymal transition and porcine embryonic brain development $(16,17)$. Recently, an increasingly number of studies have demonstrated that circRNAs can regulate gene expression by acting as microRNA sponges, such as CDRlas (18).

Of note, a growing body of evidence has indicated that circRNAs are closely associated with the pathogenesis of a number of human diseases, particularly cancer (19). To date, abnormal expression levels of circRNAs have been revealed in various types of cancer, including breast, liver, gastric, colorectal and bladder cancer (20-24). However, the characterization of circRNAs in ovarian cancer remains largely unknown. To date, and at least to the best of our knowledge, only two articles have been published in the literature regarding the association between circRNAs and ovarian cancer $(12,25)$. In the study by Ahmed et al (25), differential expression profiles of circRNAs were defined by performing paired-end RNA sequencing of primary sites, peritoneal and lymph node metastases from 3 patients with stage IIIC ovarian cancer. In the study by Bachmayr-Heyda et al (12), the expression of circular and linear RNAs and proliferation were analyzed in matched normal colon mucosa and tumor tissues. They then validated the correlation of global circular RNA abundance (the circRNA index) and the proliferation in ovarian cancer cells compared to cultured normal ovarian epithelial cells. However, neither of these two articles investigated the differential expression profiles of circRNAs in EOC specimens compared to normal ovarian tissues. In this study, we aimed to examine the pathogenesis of EOC and to identify potential therapeutic targets and circRNA biomarkers by comprehensively profiling circRNA expression in EOC specimens.

\section{Materials and methods}

Human samples. Four EOC specimens and four normal ovarian tissues for circRNA-sequencing analysis were obtained from the Department of Obstetrics and Gynecology in Peking Union Medical College Hospital (PUMCH) between 2013 and 2017. A total of 54 EOC specimens and 54 normal ovarian tissues, including those used for circRNA-sequencing analysis were collected for circRNA validation by reverse transcriptionquantitative polymerase chain reaction (RT-qPCR). Cancer specimens were collected from patients who had histologically proven to suffer from EOC and had received wedge biopsy of ovaries or cytoreductive surgery. Normal ovarian tissues were collected from patients who underwent adnexectomy due to myoma. Cancer tissues were collected from primary sites, and the normal ovarian tissues were collected from the surface epithelium. Fresh tissues were collected during surgery, frozen in liquid nitrogen within $5 \mathrm{~min}$ following resection, and stored at $-80^{\circ} \mathrm{C}$ until use. All patients were consecutive and did not receive any pre-operative chemotherapy, radiotherapy or target therapy. All the EOC specimens and normal ovarian tissues were confirmed by experienced pathologists. Clinicopathological data, including age at diagnosis, International Federation of Gynecology and Obstetrics (FIGO) stage, residual tumor diameter, lymph node metastasis (LNM), CA125 level, and ascites are presented in Table I. The study protocol conformed to the ethical guidelines of the 1975 Declaration of Helsinki, and was approved by the Ethics Committee of PUMCH. All experiments were performed in accordance with relevant guidelines. Written informed consents were obtained from all patients for research purpose.

RNA-seq analysis. Total RNA was extracted from the tissues using TRIzol reagent (Life Technologies/Thermo Fisher Scientific, Waltham, MA, USA) according to the manufacturer's instructions. Briefly, ribosomal RNA was removed from total RNA using the Epicentre Ribo-Zero rRNA Removal kit (Illumina, San Diego, CA, USA). The ribosome-depleted RNA was then treated with RNase R (Epicentre) and fragmented to approximately $200 \mathrm{bp}$. Subsequently, the purified RNA was subjected to first-strand cDNA synthesis according to the instructions provided with the NEBNext Ultra Directional RNA Library Prep kit for Illumina (NEB, Beverly, MA, USA). The cDNA fragments were repaired using an End-It DNA End Repair kit and then modified by the Klenow fragment to add an A to the 3 ' end of the DNA fragments, and were finally ligated to adapters. Purified first-strand cDNA was then subjected to 13-15 cycles of PCR amplification . The PCR protocol included an initial denaturation step $\left(98^{\circ} \mathrm{C}\right.$ for $\left.30 \mathrm{sec}\right)$ and 15 cycles of denaturation $\left(98^{\circ} \mathrm{C}\right.$ for $10 \mathrm{sec}, 65^{\circ} \mathrm{C}$ for $\left.75 \mathrm{sec}\right)$ and annealing $\left(65^{\circ} \mathrm{C}\right.$ for $\left.5 \mathrm{~min}\right)$. The library products were evaluated using the Agilent 2200 TapeStation and Qubit 2.0 (Life Technologies, Carlsbad, CA, USA) and then diluted to $10 \mathrm{pM}$ for cluster generation in situ on the HiSeq3000 pair-end flow cell followed by sequencing (2x150 bp) on HiSeq3000 (Illumina).

Identification and quantification of circRNAs. Firstly, we used TopHat2 (26) to map the RNA-seq FASTQ reads to the human reference genome (GRCh37/hg19) obtained from the UCSC genome database (http://genome.ucsc.edu/). Subsquently, as previously described, the unmapped reads were used to identify circRNAs (16). In brief, all the unmapped reads were processed to 20-nucleotide anchors from both ends of the sequencing read. Anchors aligning in the head-to-tail orientation indicated a back-spliced junction. Subsequently, the anchor alignment was extended, so that the complete read alignment and the breakpoint were flanked by a GT/AG splice site. The circRNA abundance was measured using the total number of reads that spanned back-spliced junctions. RefSeq was used to annotate the genomic regions that mapped to inferred circRNAs. Gene coordinates were obtained from the RefGene tables in the UCSC Genome Browser (downloaded on $03 / 28 / 2014$ ). A custom script was used to determine the host genes of circRNAs. The longest transcript fragment whose boundaries ( $5^{\prime}$ or $3^{\prime}$ end) exactly matched both ends of each circRNAs in the same strand was searched. The corresponding gene of this transcript fragment was then defined as the host gene of this circRNA.

$R N A$ preparation and $R T-q P C R$. Total RNA was extracted from $30-50 \mathrm{mg}$ of the EOC specimens and normal ovarian tissues using TRIzol reagent (Life Technologies/Thermo Fisher Scientific) according to the manufacturer's instructions. RT-qPCR was performed using M-MLV Reverse Transcriptase (Promega, Madison, WI, USA), PowerUp ${ }^{\text {TM }}$ SYBR $^{\text {TM }}$ Green Master Mix (Thermo Fisher Scientific) according to the manufacturer's 
instructions. GAPDH was used as an internal reference gene. The RT-qPCR protocol included an initial denaturation step $\left(95^{\circ} \mathrm{C}\right.$ for $20 \mathrm{sec})$ and 40 cycles of denaturation $\left(95^{\circ} \mathrm{C}\right.$ for $\left.3 \mathrm{sec}\right)$ and annealing $\left(60^{\circ} \mathrm{C}\right.$ for $\left.30 \mathrm{sec}\right)$. The relative expression levels were calculated using $2^{-\Delta \Delta \mathrm{Cq}}$ method (27) as previously described (28). The primer sequences were as follows: circBNC2 (forward, 5'-GCAGTTCGGAACCAGAACGAC-3' and reverse, 5'-ATG CTGGCCAGTCTTGCTCAC-3'), circEXOC6B (forward, 5'-TGC CTCAAGTAAGCCACTATC-3' and reverse, 5'-TTCCTT TCCCTCATGTTGTAG-3'), circFAM13B (forward, 5'-GTGAA AGTAACAGAGACTGTTC-3' and reverse, 5'-ACTTACTCT GTAGAGGCTGG-3'), circN4BP2L2 (forward, 5'-CATGGT GTGTCTCGAAAGAAG-3' and reverse, 5'-CTGTACCCATC TTGATGGTGA-3'), circRHOBTB3 (forward, 5'-GCTTTTCA GTGGGAAGAATTG-3' and reverse, 5'-ACATGGCTTACAGC GCAGAGA-3'), circCELSR1 (forward,5'-CGATTCGACACCAT CCATGAAG-3' and reverse, 5'-GTCCACCTGCGTCTCAT TGC-3') and GAPDH (forward, 5'-AACGTGTCAGTGGTGGAC CTG-3' and reverse, 5'-GAGACCACCTGGTGCTCAGTG-3').

Bioinformatics analysis. Gene Ontology (GO) analysis was performed to explore the functional roles of target genes in terms of biological processes, cellular components and molecular functions. Biological pathways defined by Kyoto Encyclopedia of Genes and Genomes (KEGG), Biocarta and Reactome (http://www.genome.jp/kegg/) were identified by Database for Annotation, Visualization and Integrated Discovery (DAVID; http://www.david.abcc.ncifcrf.gov/).

Statistical analysis. In the circRNA-seq analysis, circRNAs were identified as differentially expressed between the EOC specimens and normal ovarian tissues if the expression change was $\geq 2$-fold with a P-value of $<0.05$. Data from at least 3 independent experiments are expressed as the means \pm standard deviation. The Mann-Whitney U test was used to determine the statistical significance for comparisons between 2 groups, and a P-value less than $\alpha$ which was adjusted by Bonferroni correction (adjusted $\alpha$ was $0.05 / 6$, i.e., 0.0083 ) was considered statistically significant. Overall survival (OS) and progressfree survival (PFS) were calculated from the date of surgery to the date of death, progress or last follow-up (April 2018). The average follow-up time was 29.0 months (range, 4 to 52 months). Kaplan-Meier survival analysis was used for the analysis of the survival rate; The P-value was calculated by the log-rank test and a P-value $<0.05$ was considered to indicate a statistically significant difference. Statistical analyses were carried out using SPSS statistical software version 23.0 (SPSS, Chicago, IL, USA) and GraphPad Prism 5.0. All tests were two-sided.

\section{Results}

Identification of circRNAs by RNA-seq analysis in EOC. Firstly, circRNAs were characterized using the RNA-seq analysis of 4 EOC specimens and 4 normal ovarian tissues (Fig. 1A). A total of 66,521 distinct circRNA candidates were identified from these samples. Of these, 37,674 circRNAs contained at least 2 unique back-spliced reads (Fig. 1B). The circRNA candidates were annotated using the RefSeq database. More than $50 \%$ of these circRNAs were transcribed from protein-coding exons, and a small fraction of these were from introns, non-coding RNAs, 5' UTR, 3' UTR, and other sources (Fig. 1C). The median length of the exonic circRNAs was 500 nucleotides (nt) (Fig. 1D).

The relative expression analysis of these circRNAs revealed a series of differentially expressed circRNAs in the EOC specimens compared with the normal ovarian tissues (Fig. 2). Based on the RNA-seq results, circRNAs with a signal altered by 2 -fold and with value of $\mathrm{P}<0.05$ were identified as statistically altered. On the whole, 4,388 circRNAs were identified to be differentially expressed in the EOC specimens compared with the normal ovarian tissues. Of these, 2,556 circRNAs were upregulated, while 1,832 circRNAs were downregulated in the EOC specimens (data not shown).

Validation of the differential expression levels of circRNAs. In order to verify the reliability of the circRNA-seq results, the 4,388 variable expressed circRNAs were ranked based on calculating fold change and abundance, and the top 6 of the ranked circRNAs were selected for validation in the 54 EOC specimens and 54 normal ovarian tissues by RT-qPCR. Finally, 5 downregulated circRNAs and 1 upregulated circRNA were selected randomly from the 4,388 dysregulated circRNA transcripts. The results of RT-qPCR revealed that these circRNAs were significantly differentially expressed between the 2 groups. In particular, circBNC2 (hsa_circ_0008732) $(\mathrm{P}<0.0001$, Fig. 3A), circEXOC6B (hsa_circ_0009043) $(\mathrm{P}<0.0001$, Fig. 3B), circFAM13B (hsa_circ_0001535) (P<0.0001, Fig. 3C), circN4BP2L2 (hsa_circ_0000471) $(\mathrm{P}<0.0001$, Fig. 3D) and circRHOBTB3 (hsa_circ_0007444) $(\mathrm{P}<0.0001$, Fig. 3E) were significantly downregulated in the EOC specimens, whereas circCELSR1 (hsa_circ_0063809) $(\mathrm{P}<0.0001$, Fig. 3F) was significantly upregulated in the EOC group (Fig. 4). The results of qRT-PCR were consistent with the circRNA-seq results, which further verified the reliability of our circRNA-seq results.

The association between circRNAs and the clinicopathological features of EOC. The association between circRNAs and various clinicopathological features of EOC was evaluated in the 54 patients with EOC. The median expression levels of circRNAs were used to divide the circRNAs into the high level group and the low level group. Our data revealed that the low level of circBNC2 was associated with younger age $(\mathrm{P}=0.027)$, an advanced FIGO stage $(\mathrm{P}=0.045)$, LNM $(\mathrm{P}=0.005)$ and massive ascites $(\mathrm{P}=0.034)$. The low level of circEXOC6B was associated with younger age $(\mathrm{P}=0.018)$ and $\mathrm{LNM}(\mathrm{P}=0.032)$. The low level of circFAM13B was associated with younger age $(\mathrm{P}=0.032)$, an advanced FIGO stage $(\mathrm{P}=0.044)$ and LNM $(\mathrm{P}=0.034)$. The low level of circN4BP2L2 was associated with younger age $(\mathrm{P}=0.047)$, an advanced FIGO stage $(\mathrm{P}=0.046)$ and LNM $(\mathrm{P}=0.003)$. The low level of circRHOBTB3 was associated with younger age $(\mathrm{P}=0.023)$ and $\mathrm{LNM}(\mathrm{P}=0.009)$. In addition, the high level of circCELSR1 was associated with an advanced FIGO stage $(\mathrm{P}=0.043)$, a larger residual tumor diameter $(\mathrm{P}=0.035)$ and massive ascites $(\mathrm{P}=0.021)$ (Table $\mathrm{I})$.

Prognostic value of circRNAs in EOC. We further investigated the prognostic value of various clinicopathological features and differentially expressed circRNAs in patients with EOC. The results from univariate analysis revealed that, 


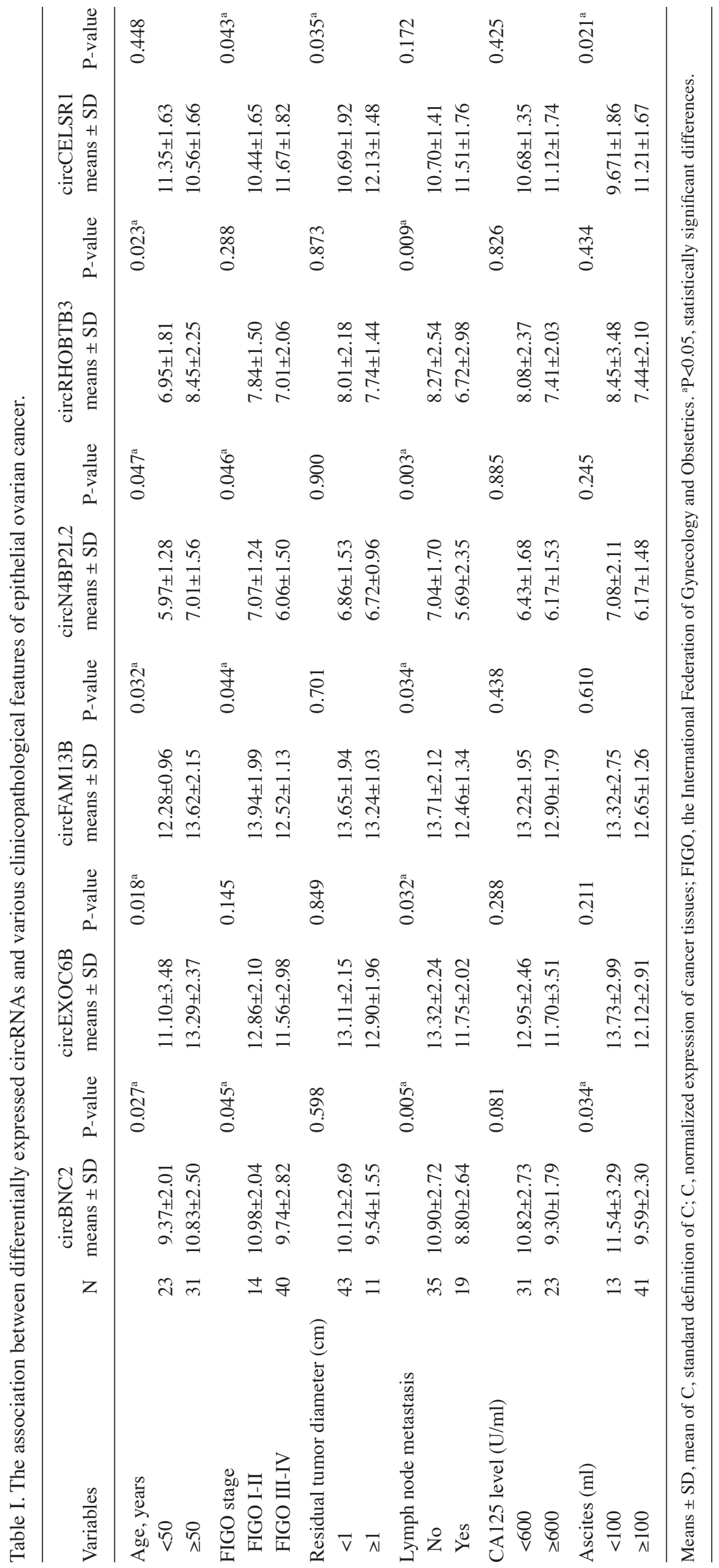


A

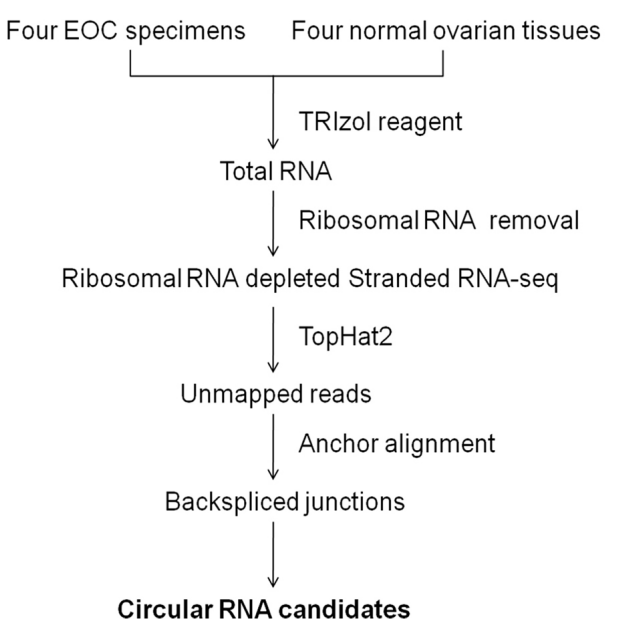

C

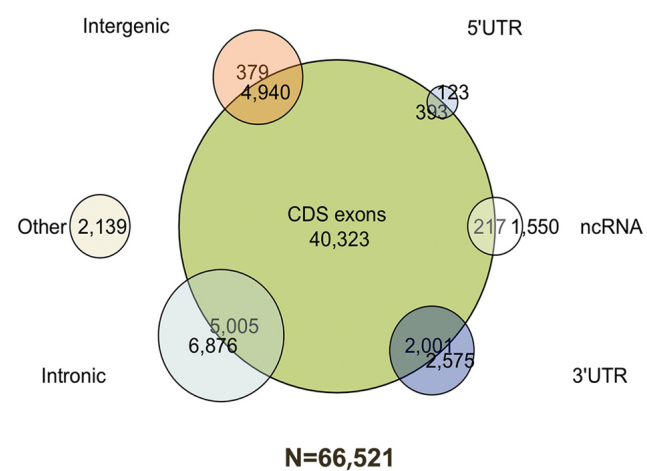

B
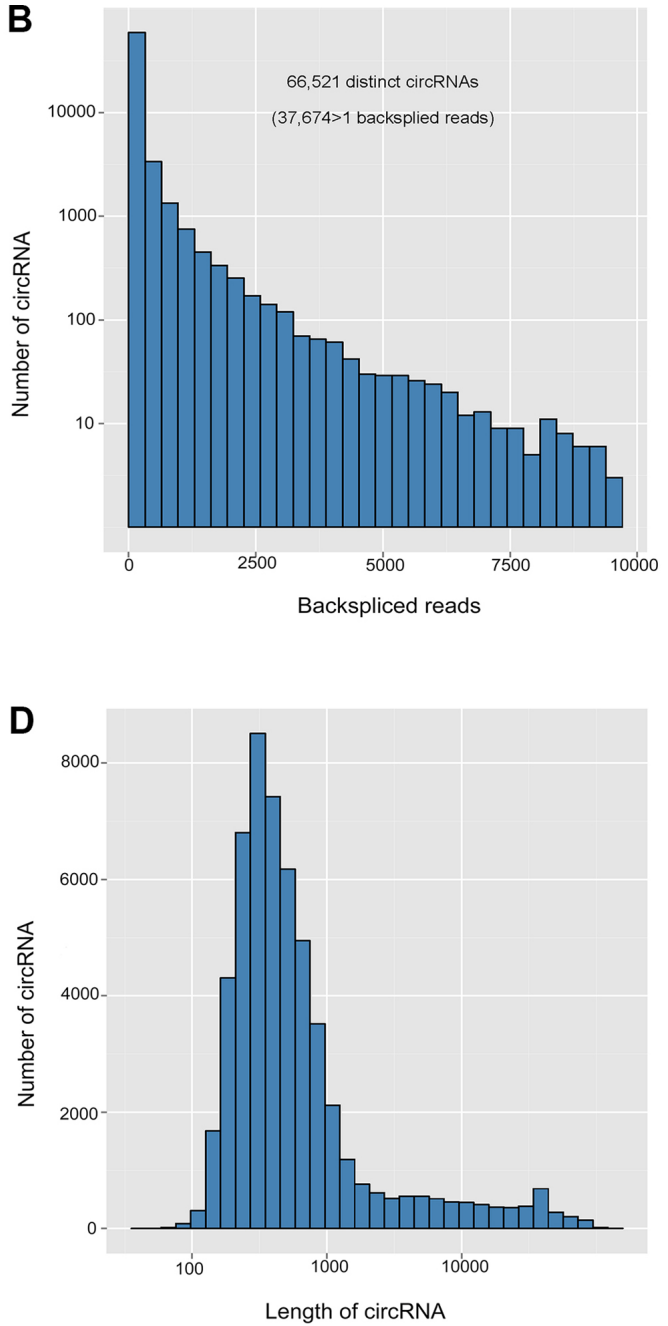

Figure 1. Profiling of circular RNAs (circRNAs) in human epithelial ovarian cancer (EOC) specimens and normal ovarian tissues. (A) circRNA-sequencing analysis in 4 EOC specimens and 4 normal ovarian tissues. (B) The total number of circRNAs and back-spliced reads identified in 4 EOC specimens and 4 normal ovarian tissues. (C) Genomic origin of circRNAs. (D) The length distribution for exonic circRNAs (n=54,351, only known spliced length was considered).

an advanced FIGO stage $(\mathrm{P}=0.003$, log-rank test $)$ and LNM $(\mathrm{P}=0.024$, log-rank test) were predictive of a worse PFS. Moreover, a high level of circN4BP2L2 ( $\mathrm{P}=0.025$, log-rank test) was associated with a better PFS. In addition, a high level of circEXOC6B $(\mathrm{P}=0.045$, log-rank test) was associated with a better OS (Table II).

Bioinformatics analyses. The results of GO enrichment analysis of the differentially expressed circRNAs with identified target genes are shown in Fig. 5. GO analysis revealed that numerous target genes were involved in the biological processes, such as cellular process, regulation of biological process, metabolic process, etc. These processes were associated with human tumorigenesis and metastasis. KEGG analysis revealed that there were 23 pathways related to differentially expressed circRNAs, including the ErbB signaling pathway, ECM-receptor interaction and focal adhesion (Fig. 6).

\section{Discussion}

In this study, we successfully identified a large number of circRNAs in EOC specimens. We also found that a considerable portion of these circRNAs were differentially expressed in cancer specimens compared with normal ovarian tissues. Our findings suggest that these differentially expressed circRNAs may be regulated and may play an important role in the development of EOC. We further evaluated the prognostic value of circRNAs in EOC and found that circEXOC6B and circN4BP2L2 may have potential for use as prognostic biomarkers in EOC. To the best of our knowledge, we are the first to perform a detailed circRNA-seq analysis of EOC species and normal ovarian tissues.

The circNRA expression profiles in this study demonstrated that 2,556 circRNAs were abnormally upregulated and 1,832 circRNAs were downregulated in the EOC specimens compared with the normal ovarian tissues. In particular, circBNC2, circEXOC6B, circFAM13B, $\operatorname{circN} 4 \mathrm{BP} 2 \mathrm{~L} 2$, circRHOBTB3 and circCELSR1 were validated to be significantly dysregulated in the EOC specimens compared with the normal ovarian tissues by RT-qPCR. Recently, Bahn et al (26) successfully identified $>400$ circRNAs in human cell-free saliva. It has also been shown that, compared to their corresponding linear counterparts, circRNAs have a higher expression levels in blood. While the liner mRNAs 


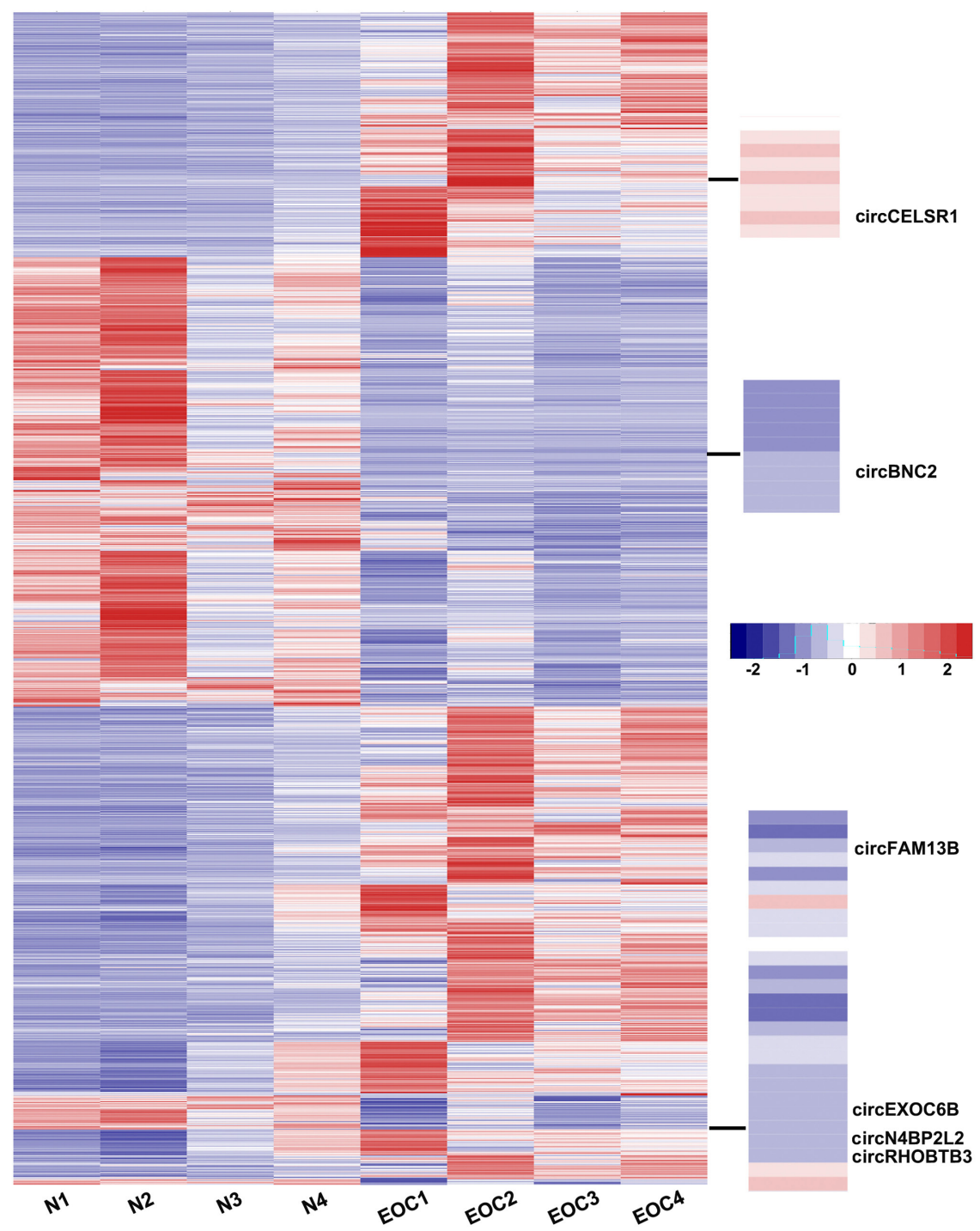

Figure 2. Clustered heatmap of the differentially expressed circular RNAs (circRNAs) in 4 epithelial ovarian cancer (EOC) specimens and 4 normal ovarian tissues. Columns represent tissues, while rows represent circRNAs.
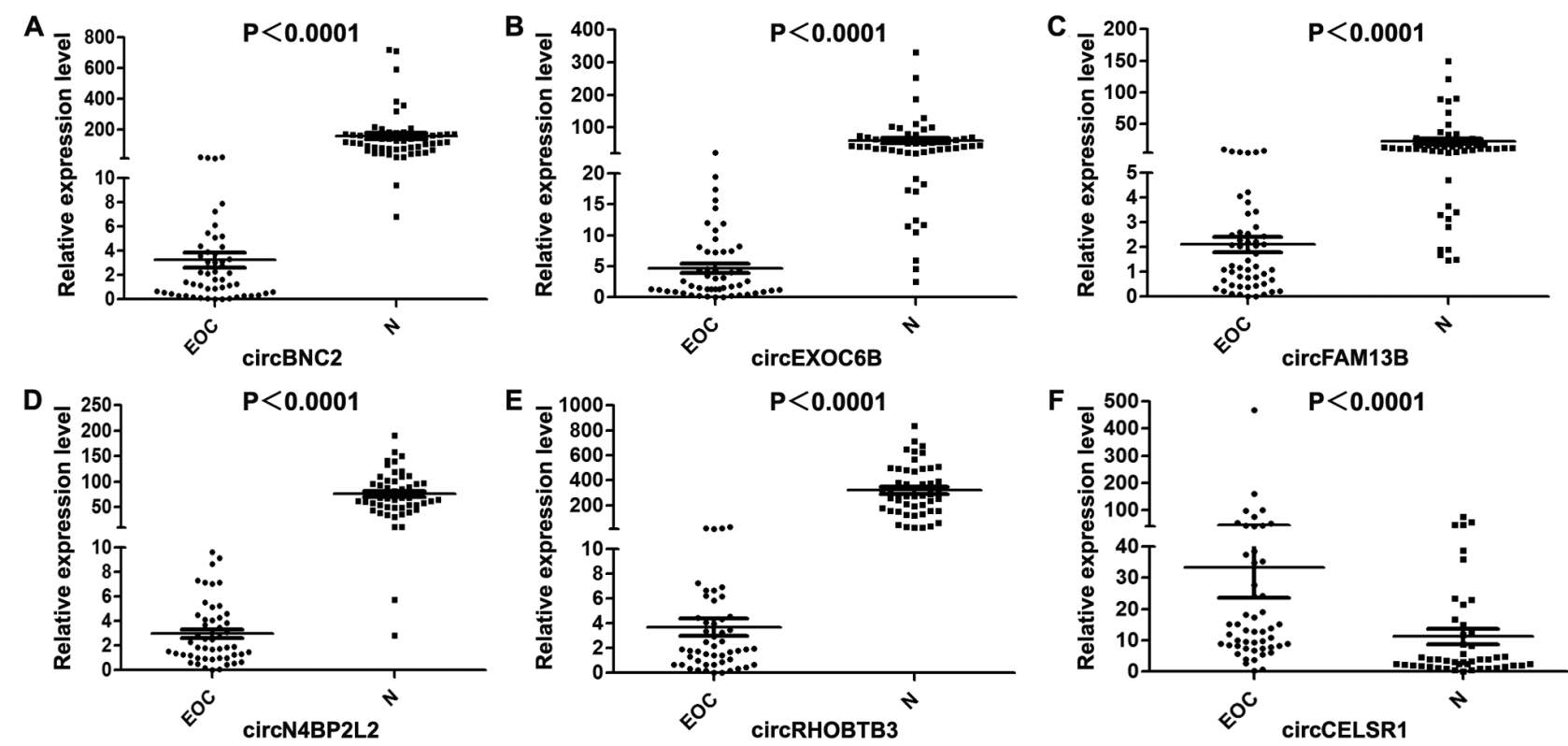

Figure 3. Validation of 6 differentially expressed circular RNA (circRNA) candidates in 54 epithelial ovarian cancer (EOC) specimens and 54 normal ovarian tissues by RT-qPCR. (A) circBNC2, (B) circEXOC6B, (C) circFAM13B, (D) circN4BP2L2, (E) circRHOBTB3 and (F) circCELSR1. 


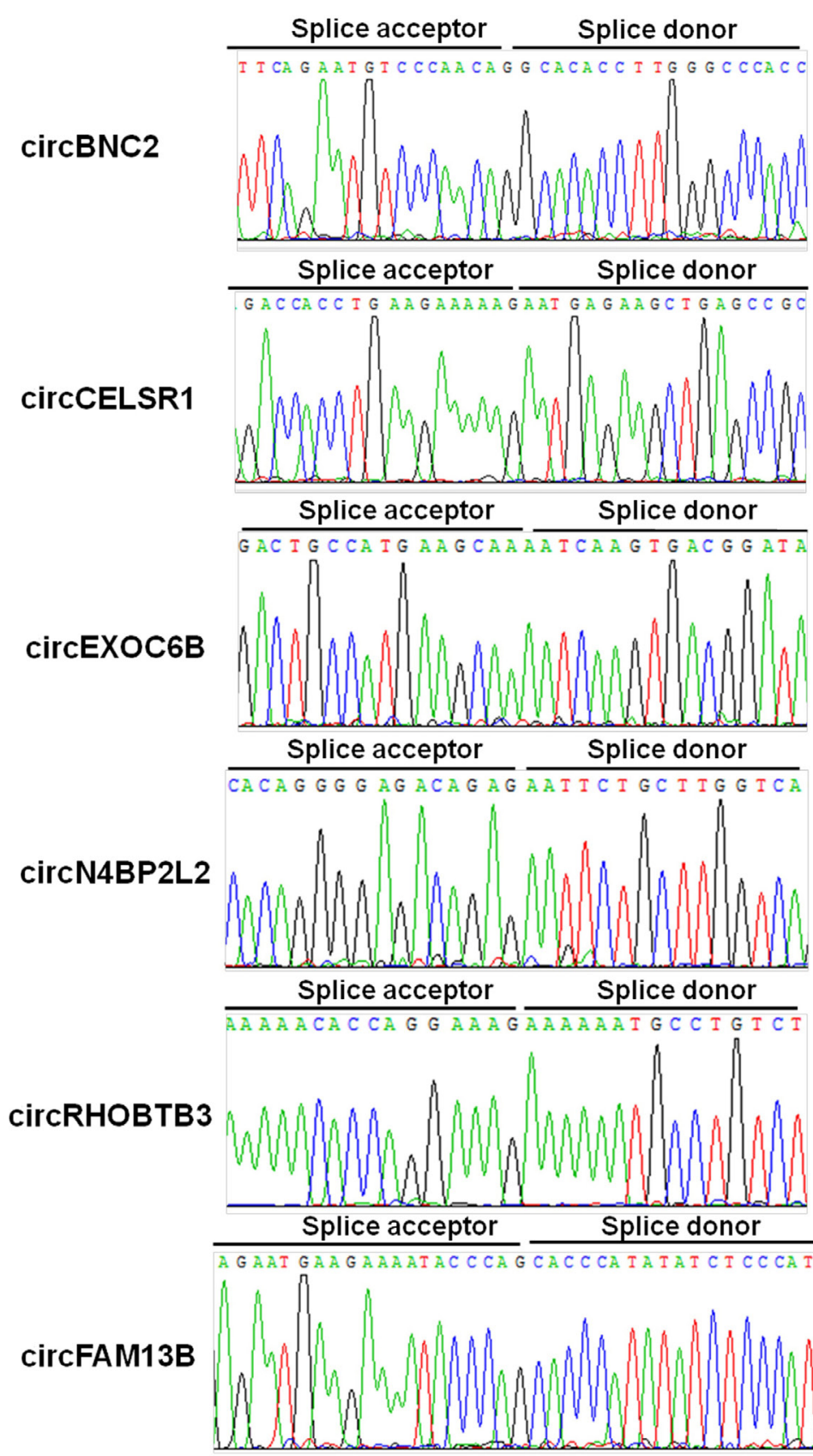

Figure 4. circBNC2, circCELSR1, circEXOC6B, circN4BP2L2, circRHOBTB3 and circFAM13B were validated by sanger sequencing. Splice donor and splice acceptor are indicated.

are actually absent, the circRNA isoforms are easily detected, thus enabling circRNAs to embody disease information which conventional RNA assay fails to determine (19). Given the abundance of circRNAs, their tissue-specific expression patterns and their high stability in vivo, circRNAs may be used as promising biomarkers for clinical applications in the future. Accordingly, the results of this study suggest that circBNC2, circEXOC6B, circFAM13B, circN4BP2L2, circRHOBTB3 and circCELSR1 may be potential diagnostic biomarkers for EOC. We further tested these 6 circRNAs in our laboratory with tissues and plasma.

By performing outcome analysis, we found that the expression levels of circEXOC6B and circN4BP2L2 were significantly associated with OS and PFS, respectively. The OS of patients with EOC with a low circEXOC6B expression was significantly worse than that of patients with a high circEXOC6B expression. Moreover, patients with EOC with a low circN4BP2L2 expression had a significantly worse PFS than those with a high circN4BP2L2 expression. These results suggested that circEXOC6B and circN4BP2L2 may act as important and useful prognostic biomarkers in patients with EOC. Our data demonstrated that circEXOC6B and circN4BP2L2 were negatively associated with FIGO stage and/ or LNM. In addition, we found that FIGO stage and LNM were negatively associated with the survival of patients with EOC. Thus, circEXOC6B and circN4BP2L2 may play a profound role in regulating tumor invasion and metastasis. Prognostic biomarkers can help in selecting personalized treatment regimens for patients with EOC. circRNAs are highly stable in cells, and their tissue-specific character make them more 
Table II. The prognostic values of various clinicopathological features and differentially expressed circRNAs in patients with epithelial ovarian cancer.

\begin{tabular}{|c|c|c|c|c|c|}
\hline Variables & $\mathrm{N}$ & $\begin{array}{c}\text { OS } \\
\text { Rate }(\%)\end{array}$ & P-value & $\begin{array}{c}\text { PFS } \\
\text { Rate }(\%)\end{array}$ & P-value \\
\hline \multicolumn{6}{|c|}{ All cases $(n=54)$} \\
\hline \multicolumn{6}{|c|}{ Age (years) } \\
\hline$<50$ & 23 & 69.6 & \multirow[t]{2}{*}{0.526} & 39.1 & \multirow[t]{2}{*}{0.334} \\
\hline$\geq 50$ & 31 & 80.6 & & 51.6 & \\
\hline \multicolumn{6}{|c|}{ FIGO stage } \\
\hline I-II & 14 & 92.9 & \multirow[t]{2}{*}{0.13} & 78.6 & \multirow[t]{2}{*}{$0.003^{\mathrm{a}}$} \\
\hline III-VI & 40 & 70.0 & & 35.0 & \\
\hline \multicolumn{6}{|c|}{ Residual tumor diameter $(\mathrm{cm})$} \\
\hline$<1$ & 43 & 74.4 & \multirow[t]{2}{*}{0.866} & 44.2 & \multirow[t]{2}{*}{0.570} \\
\hline$\geq 1$ & 11 & 81.8 & & 54.5 & \\
\hline \multicolumn{6}{|c|}{ Lymph node metastasis } \\
\hline No & 35 & 80.0 & \multirow[t]{2}{*}{0.354} & 60.0 & \multirow[t]{2}{*}{$0.024^{\mathrm{a}}$} \\
\hline Yes & 19 & 63.2 & & 21.1 & \\
\hline \multicolumn{6}{|c|}{ CA125 level (U/ml) } \\
\hline$<600$ & 31 & 74.2 & \multirow[t]{2}{*}{0.817} & 48.4 & \multirow[t]{2}{*}{0.860} \\
\hline$\geq 600$ & 23 & 78.3 & & 43.5 & \\
\hline \multicolumn{6}{|c|}{ Ascites (ml) } \\
\hline$<100$ & 13 & 76.9 & \multirow[t]{2}{*}{0.661} & 53.8 & \multirow[t]{2}{*}{0.350} \\
\hline$\geq 100$ & 41 & 75.6 & & 43.9 & \\
\hline \multicolumn{6}{|l|}{$\operatorname{circBNC2}$} \\
\hline High & 27 & 70.4 & \multirow[t]{2}{*}{0.814} & 51.9 & \multirow[t]{2}{*}{0.809} \\
\hline Low & 27 & 81.5 & & 40.7 & \\
\hline \multicolumn{6}{|c|}{ circEXOC6B } \\
\hline High & 27 & 88.9 & \multirow[t]{2}{*}{$0.045^{\mathrm{a}}$} & 48.1 & \multirow[t]{2}{*}{0.636} \\
\hline Low & 27 & 63.0 & & 44.4 & \\
\hline \multicolumn{6}{|c|}{ circFAM13B } \\
\hline High & 27 & 77.8 & \multirow[t]{2}{*}{0.956} & 48.1 & 0.939 \\
\hline Low & 27 & 74.1 & & 44.4 & \\
\hline circN4BP2 & & & & & \\
\hline High & 27 & 85.2 & 0.059 & 59.3 & $0.025^{\mathrm{a}}$ \\
\hline Low & 27 & 66.7 & & 33.3 & \\
\hline circRHOB & & & & & \\
\hline High & 27 & 81.5 & 0.052 & 51.9 & 0.121 \\
\hline Low & 27 & 70.4 & & 40.7 & \\
\hline circCELSF & & & & & \\
\hline High & 27 & 74.1 & 0.856 & 44.4 & 0.944 \\
\hline Low & 27 & 77.8 & & 48.1 & \\
\hline
\end{tabular}

circRNAs, circular RNAs; FIGO, the International Federation of Gynecology and Obstetrics; OS, overall survival; PFS, progress-free survival. ${ }^{a} \mathrm{P}<0.05$, statistically significant differences.

sensitive to reflect the real condition in vivo (6). Therefore, our findings verified the promising clinical values of circRNAs in tumor diagnosis and treatment.

This study has some limitations. Since the sample size in our study was relatively small and was limited to Han ethnicity Chinese patients from a single institution, the specificity and accuracy of the model cannot be estimated accurately. Therefore, prospectively multicenter studies with a larger sample size and heterogeneous cohorts of patients should be conducted in the future to verify our results. Furthermore, functional experimental analyses of cancer cell lines and experimental animals should be further conducted to 


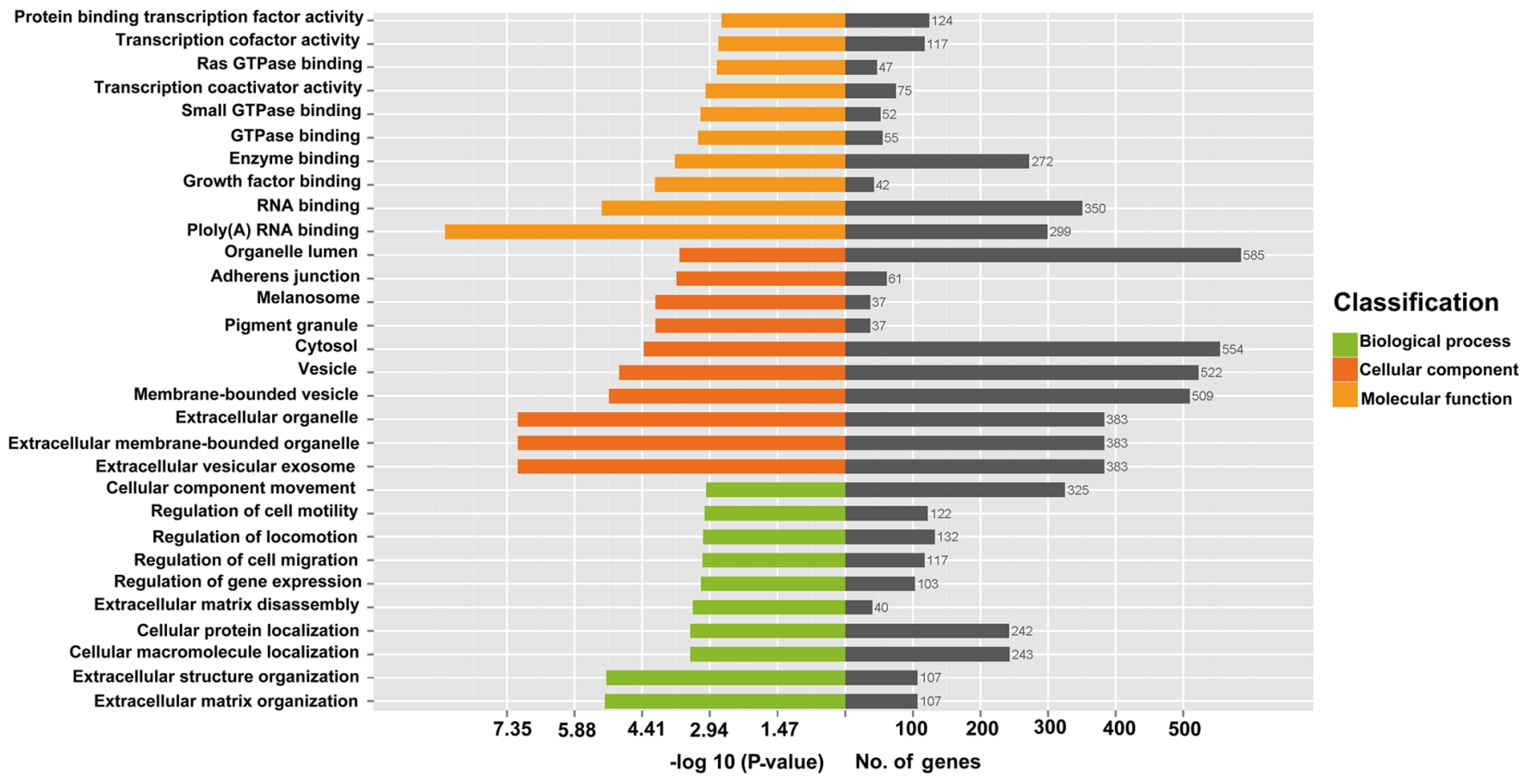

Figure 5. Gene Ontology enrichment analysis on the differentially expressed circular RNAs (circRNAs) with identified target genes.

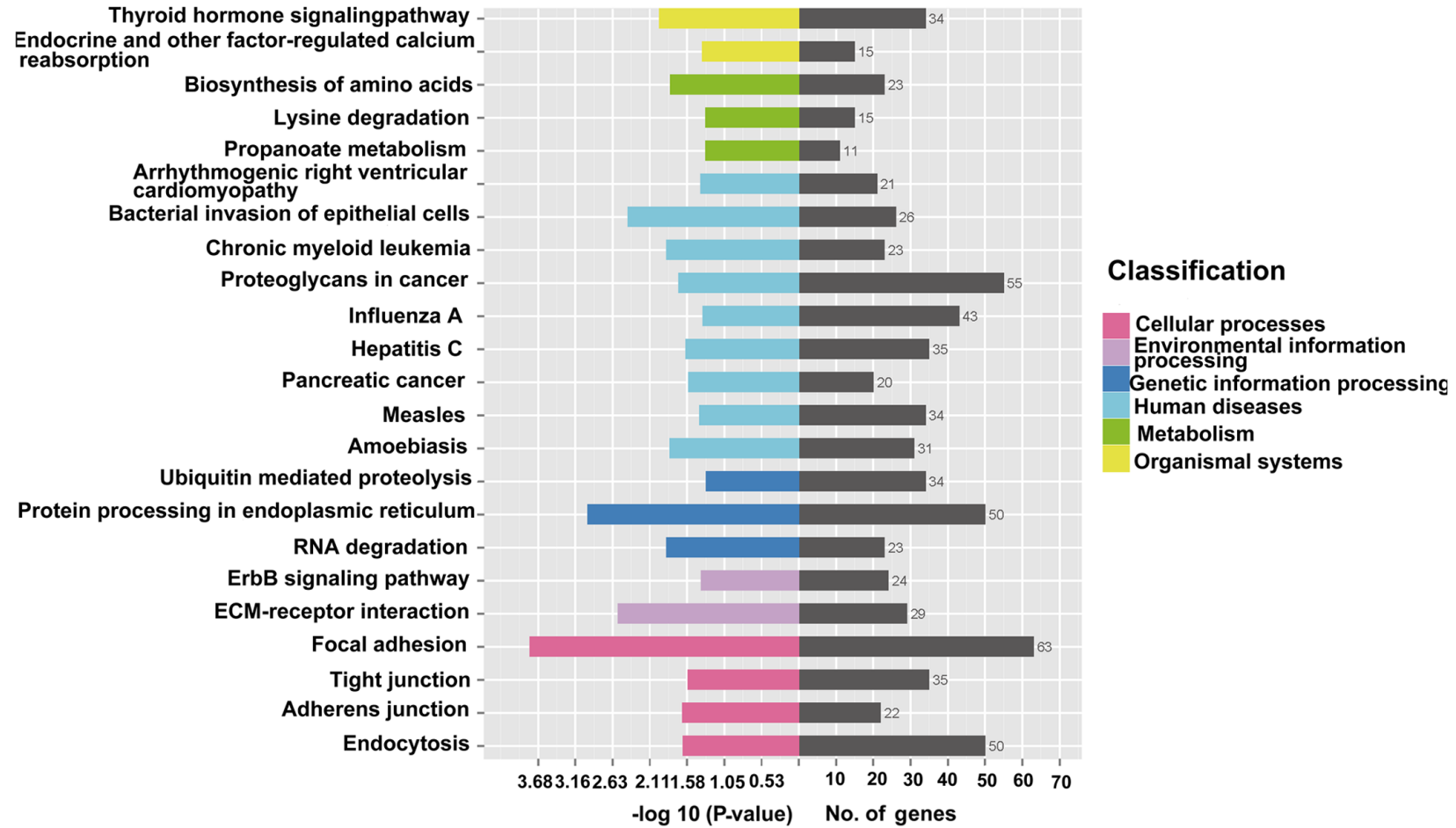

Figure 6. KEGG analysis revealed that there were 23 pathways related to differentially expressed circular RNAs (circRNAs).

determine whether these circRNAs are involved in the tumorigenesis and progression of EOC.

In conclusion, this study identified the circRNA profile of EOC specimens. We demonstrated that circBNC2, circEXOC6B, circFAM13B, circN4BP2L2, circRHOBTB3 and circCELSR1 were significantly dysregulated in EOC specimens and may be potential diagnostic biomarkers for EOC. We also found that circEXOC6B and circN4BP2L2 may serve as novel prognostic biomarkers in patients with EOC. However, future experiments with a larger sample are required to verify the current findings and to elucidate the regulatory mechanisms of action of circRNAs in the tumorigenesis of EOC.

\section{Acknowledgements}

Not applicable. 


\section{Funding}

This study was funded by the Chinese Academy of Medical Sciences Initiative for Innovative Medicine (grant no. CAMS-2017-I2M-1-002).

\section{Availability of data and materials}

The datasets used and/or analyzed during the current study are available from the corresponding author on reasonable request.

\section{Authors' contributions}

LN, BL, YH and JHL contributed to study conception and design, data analysis, and manuscript preparation. WZ contributed to data analysis. MY, SW, DC, JY and KS contributed to data collection and data analysis. All authors have read and approved the final version of the manuscript.

\section{Ethics approval and consent to participate}

The use of human tissues was approved by the Ethics Committee of PUMCH and patient consent was obtained.

\section{Consent for publication}

Not applicable.

\section{Competing Interests}

The authors declared that they have no competing interests.

\section{References}

1. Torre LA, Bray F, Siegel RL, Ferlay J, Lortet-Tieulent J and Jemal A: Global cancer statistics, 2012. CA Cancer J Clin 65: 87-108, 2015.

2. Siegel RL, Miller KD and Jemal A: Cancer statistics, 2016. CA Cancer J Clin 66: 7-30, 2016.

3. Bowtell DD, Böhm S, Ahmed AA, Aspuria PJ, Bast RC Jr, Beral V, Berek JS, Birrer MJ, Blagden S, Bookman MA, et al: Rethinking ovarian cancer II: Reducing mortality from highgrade serous ovarian cancer. Nat Rev Cancer 15: 668-679, 2015.

4. Kroeger PT Jr and Drapkin R: Pathogenesis and heterogeneity of ovarian cancer. Curr Opin Obstet Gynecol 29: 26-34, 2017.

5. Jeck WR and Sharpless NE: Detecting and characterizing circular RNAs. Nat Biotechnol 32: 453-461, 2014.

6. Wang PL, Bao Y, Yee MC, Barrett SP, Hogan GJ, Olsen MN, Dinneny JR, Brown PO and Salzman J: Circular RNA is expressed across the eukaryotic tree of life. PLoS One 9: e90859, 2014.

7. Cocquerelle C, Mascrez B, Hétuin D and Bailleul B: Mis-splicing yields circular RNA molecules. FASEB J 7: 155-160, 1993.

8. Salzman J, Gawad C, Wang PL, Lacayo N and Brown PO: Circular RNAs are the predominant transcript isoform from hundreds of human genes in diverse cell types. PLoS One 7: e30733, 2012.

9. Lasda E and Parker R: Circular RNAs: Diversity of form and function. RNA 20: 1829-1842, 2014.
10. Starke S, Jost I, Rossbach O, Schneider T, Schreiner S, Hung LH and Bindereif A: Exon circularization requires canonical splice signals. Cell Rep 10: 103-111, 2015.

11. Ashwal-Fluss R, Meyer M, Pamudurti NR, Ivanov A, Bartok O, Hanan M, Evantal N, Memczak S, Rajewsky N and Kadener S: circRNA biogenesis competes with pre-mRNA splicing. Mol Cell 56: 55-66, 2014.

12. Bachmayr-Heyda A, Reiner AT, Auer K, Sukhbaatar N, Aust S, Bachleitner-Hofmann T, Mesteri I, Grunt TW, Zeillinger R and Pils D: Correlation of circular RNA abundance with proliferation - exemplified with colorectal and ovarian cancer, idiopathic lung fibrosis, and normal human tissues. Sci Rep 5: 8057, 2015.

13. Jeck WR, Sorrentino JA, Wang K, Slevin MK, Burd CE, Liu J, Marzluff WF and Sharpless NE: Circular RNAs are abundant, conserved, and associated with ALU repeats. RNA 19: 141-157, 2013.

14. Chen LL and Yang L: Regulation of circRNA biogenesis. RNA Biol 12: 381-388, 2015.

15. Li Y, Zheng Q, Bao C, Li S, Guo W, Zhao J, Chen D, Gu J, He X and Huang S: Circular RNA is enriched and stable in exosomes: A promising biomarker for cancer diagnosis. Cell Res 25: 981-984, 2015.

16. Conn SJ, Pillman KA, Toubia J, Conn VM, Salmanidis M, Phillips CA, Roslan S, Schreiber AW, Gregory PA and Goodall GJ: The RNA binding protein quaking regulates formation of circRNAs. Cell 160: 1125-1134, 2015.

17. Ven $\varnothing$ MT, Hansen TB, Ven $\varnothing$ ST, Clausen BH, Grebing M, Finsen B, Holm IE and Kjems J: Spatio-temporal regulation of circular RNA expression during porcine embryonic brain development. Genome Biol 16: 245, 2015.

18. Yu L, Gong X, Sun L, Zhou Q, Lu B and Zhu L: The circular RNA Cdrlas act as an oncogene in hepatocellular carcinoma through targeting miR-7 expression. PLoS One 11: e0158347, 2016.

19. Chen Y, Li C, Tan C and Liu X: Circular RNAs: A new frontier in the study of human diseases. J Med Genet 53: 359-365, 2016.

20. Nair AA, Niu N, Tang X, Thompson KJ, Wang L, Kocher JP, Subramanian S and Kalari KR: Circular RNAs and their associations with breast cancer subtypes. Oncotarget 7: 80967-80979, 2016.

21. Shang X,LiG,LiuH,Li T,LiuJ,ZhaoQandWang C:Comprehensive circular RNA profiling reveals that hsa_circ_0005075, a new circular RNA biomarker, is involved in hepatocellular carcinoma development. Medicine (Baltimore) 95: e3811, 2016.

22. Chen J, Li Y, Zheng Q, Bao C, He J, Chen B, Lyu D, Zheng B, $\mathrm{Xu} \mathrm{Y}$, Long Z, et al: Circular RNA profile identifies circPVT1 as a proliferative factor and prognostic marker in gastric cancer. Cancer Lett 388: 208-219, 2017.

23. Zhu M, Xu Y, Chen Y and Yan F: Circular BANP, an upregulated circular RNA that modulates cell proliferation in colorectal cancer. Biomed Pharmacother 88: 138-144, 2017.

24. Zhong Z, Lv M and Chen J: Screening differential circular RNA expression profiles reveals the regulatory role of circTCF25miR-103a-3p/miR-107-CDK6 pathway in bladder carcinoma. Sci Rep 6: 30919, 2016.

25. Ahmed I,Karedath T, Andrews SS, Al-Azwani IK, Mohamoud YA, Querleu D, Rafii A and Malek JA: Altered expression pattern of circular RNAs in primary and metastatic sites of epithelial ovarian carcinoma. Oncotarget 7: 36366-36381, 2016.

26. Bahn JH, Zhang Q, Li F, Chan TM, Lin X, Kim Y, Wong DT and Xiao X: The landscape of microRNA, Piwi-interacting RNA, and circular RNA in human saliva. Clin Chem 61: 221-230, 2015.

27. Livak KJ and Schmittgen TD: Analysis of relative gene expression data using real-time quantitative PCR and the 2(-Delta Delta C(T)) method. Methods 25: 402-408, 2001.

28. Wang P and Lv L: miR-26a induced the suppression of tumor growth of cholangiocarcinoma via KRT19 approach. Oncotarget 7: 81367-81376, 2016. 